\title{
Plasmon-Enhanced Electrocatalytic Oxygen Reduction in Alkaline Media on Gold Nanohole Electrodes ${ }^{\dagger}$
}

Received 00th January 20xx, Accepted 00th January 20xx

DOI: $10.1039 / x 0 x \times 00000 x$

\author{
Tamazouzt Nait Saada, ${ }^{\mathrm{a}, \mathrm{b}}$ Anderson Gabriel Marques da Silva, ${ }^{\mathrm{a}, \mathrm{c}, \mathrm{d}}$ Palaniappan Subramanian, ${ }^{\mathrm{e}}$ Liuqing

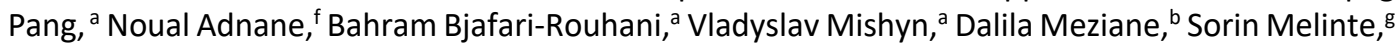 \\ Georgiana Sandu, ${ }^{\mathrm{g}}$ Franck Dumeignil, ${ }^{\mathrm{c}}$ Sébastien Paul, ${ }^{\mathrm{c}}$ Robert Wojcieszak, ${ }^{\mathrm{c}}$ Rabah Boukherroub, ${ }^{\mathrm{a}}$ \\ Sabine Szunerits ${ }^{{ }^{*}}$
}

\section{Introduction}

A great deal of research attention has been lately put into the development of alternative energy conversion technologies including, next to metal-air batteries and supercapacitors, fuel cells and electrolyzers. Electrochemical oxygen reactions, namely the oxygen reduction reaction (ORR) and oxygen evolution reaction, govern the energy transfer efficiency in metal-air batteries and fuel cells. ${ }^{1,2}$ The sluggish kinetics of ORR and the complex four-electron transfer process have been recognized as barriers for the construction of high-quality fuel cells and metal-air batteries. Indeed, oxygen reduction via a four-electron pathway competes with the production of undesired hydrogen peroxide in a two-electron transfer pathway. Any improvement in suppressing the undesired intermediate along with the enhnacement in the performance of the ORR kinetics are highly sought after. ${ }^{2}$ Conventional platinum-based materials are considered as the state-of-the-art catalysts for ORR. ${ }^{3}$ Their scarcity, elevated costs and limited

a. Univ. Lille, CNRS, Centrale Lille, ISEN, Univ. Valenciennes, UMR 8520 - IEMN, F59000 Lille, France

b. Laboratory of Applied Chemistry and Chemical Engineering (LCAGC), Université Mouloud Mammeri de Tizi Ouzou. Algeria.

Univ. Lille, CNRS, Centrale Lille, ENSCL, Univ. Artois, UMR 8181 - UCCS - Unité de Catalyse et Chimie du Solide, F-59000 Lille, France

d. Institute of Chemistry, University of São Paulo, São Paulo, Brazil

e. Department of Materials Engineering, KU Leuven, Kateelpark Arenberg, Heverlee - 3001, Belgium

f. LPMR, Faculté Pluridisciplinaire Nador, Université Mohamed Premier, 60000 Oujda, Morocco

g.7Institute of Information and Communication Technologies, Electronics and Applied Mathematics, Université catholique de Louvain, 1348 Louvain-la-Neuve, Belgium

h. Electronic Supplementary Information (ESI) available: [details of any supplementary information available should be included here]. See DOI: $10.1039 / x 0 x \times 00000 x$ long-term electrochemical activity restrict their large-scale utilization and call for the search of alternative electrocatalytic materials.

Most of the research efforts in improving the kinetics of ORR have been devoted to the synthesis of metal catalysts with optimal binding energy and high surface area that can support efficient reduction of oxygen to water. ${ }^{4} \mathrm{~A}$ very interesting opportunity to improve electrochemical oxygen reduction reaction comes from plasmon-mediated photo-electrochemical water splitting ${ }^{1}$ and plasmon-mediated electrocatalytic oxygen reduciton ${ }^{5}$ Essentially, the activation energy required to break the strong chemical bonds in water can be tuned via excitation of nanostructures that support localized surface plasmon resonance (LSPR) modes. The key role of plasmons in ORR electrocatalysis was demonstrated in recent studies.6,7 Shi et al. showed the interest of $\mathrm{Ag}$ nanoparticles/graphene electrocatalysts for plasmon-mediated ORR. ${ }^{6}$ Lin et al. gave a first empirical evidence of suppressing the formation of undesired peroxide intermediate through plasmonic effects using Ag-Pt bimetallic nanocages with an edgeless feature. ${ }^{7}$

Surprisingly, there are only a few reports on plasmon-enhanced electrochemical reactions using the intertwined plasmoncatalytic-electrochemical properties of gold nanostructures. ${ }^{8-10}$. Minteer and co-workers fabricated $\mathrm{Au}$ nanofiber-based electrode for plasmon-enhanced electrocatalytic oxidation of ethanol and methanol in alkaline media. ${ }^{8}$ Gold nanoparticles (NPs) deposited on glassy carbon electrodes (Au NPs/GCEs) were used for plasmon-accelerated electrochemical conversion of glucose to acetic acid. Wang et al. shed light onto the importance of the shape of the gold nanostructures, by revealing that plasmonic triangular nanoplates perform better in plasmon-accelerating oxidation of ascorbic acid due to the 
increased number for "hot spots" in these gold nanostructures. ${ }^{10}$

In this study, we report the exceptional plasmon-promoted properties of an array of gold nanoholes (Au NHs) for the electrochemical reduction of oxygen in alkaline electrolytes. Next to being an outstanding light absorbing material, the excellent conducting properties of $\mathrm{Au} \mathrm{NHs}$ make them well suited in electrochemical setups. We highlight here that these plasmonic electrodes are exhibiting ORR activity comparable to that of $\mathrm{Pt} / \mathrm{C}$ catalysts in alkaline media. We further demonstrate that plasmon-mediated ORR carried out on $\mathrm{Au} \mathrm{NHs}$ electrodes facilitates direct reduction of oxygen to water, thus avoiding the undesirable hydrogen peroxide intermediate.

\section{Results and discussion}

\subsection{Plasmon-enhanced charge transfer on nanoperforated gold thin film electrodes}

\subsubsection{Plasmonic component}

Plasmon-enhanced electrochemistry was performed on nanoperforated gold thin films. The samples (S1) were formed using colloidal lithography on Kapton (K) and support different LSPR modes related to holes of $630 \pm 10 \mathrm{~nm}$ diameter with a center-to-center spacing $a=980 \mathrm{~nm}$ (Fig. 1a). Elemental mapping at the microstructural level by scanning electron microscopy (SEM) with energy dispersive X-ray spectrometry (EDS) (Fig. 1b) reveals the formation of a perforated gold thin film. The ultraviolet-visible (UV/vis) absorption spectrum displays two obvious plasmon bands at 670 and $980 \mathrm{~nm}$ due to the excitation of surface plasmon waves in S1, acting as twodimensional diffraction gratings that convert incident photons into SP waves (Fig. 1c). ${ }^{11}$ The increase in absorption $<500 \mathrm{~nm}$ is due to the underlying Kapton substrate showing strong absorption at this wavelength range.

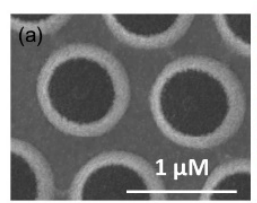

(d)

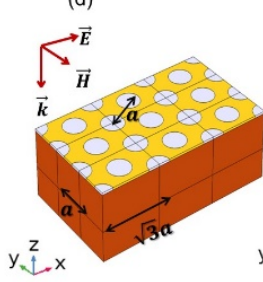

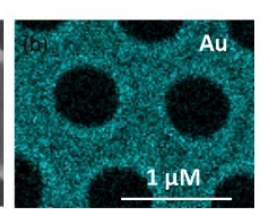

(e)

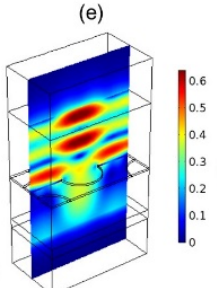

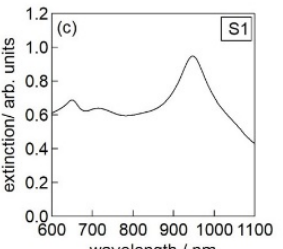

(f)

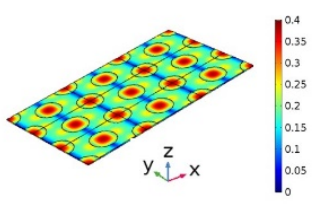

Fig 1. Plasmonic characteristics of the electrodes: (a) Scanning electron microscopy (SEM) image of a nano-perforated gold thin film electrode (S1); (b) EDS mapping of Au, (c) UV/vis absorption spectrum of S1; (d) Sketch of the simulated structure consisting of an $\mathrm{Au} \mathrm{NHs}$ hexagonal grating deposited on a semi-infinite Kapton substrate. Incidence medium (air) is set on top of the Au NHs. (e) Computed electric field map depicted in the zx plane; (e) Computed electric field map depicted in the xy plane. The scale bars consider a normalized electric field in S1 with respect to the maximum value of the field taken in some points of the system.

To get insight into the surface plasmon modes of S1, the electric field distribution within the structure was determined using a finite element method. A sketch of the S1 cell used in the numerical computations is seen in Fig. 1d and the corresponding results are shown in Fig. 1e-f. The simulated electric field distribution within the structure for an incident light wave at $980 \mathrm{~nm}$ indicates the excitation of a surface plasmon mode of S1. One notes in particular the enhancement of the electric field inside the nanoholes (Fig. 1d), which is characteristic of a surface plasmon mode.

Fig. 2a shows the temperature distribution map within a zx-cut plane (in the middle of the structure) at different instants of time: $t=1.7 \mu \mathrm{s}$ (near $\mathrm{t}=0$ ), $\mathrm{t}=5 \mu \mathrm{s}$ and $\mathrm{t}=20 \mu \mathrm{s}$ (at equilibrium). Plasmon driven heating starts off within the metal film part near and around the nanoholes where the localized plasmon is oscillating. The generated heat diffuses towards the upper boundary (water surface in contact with air) playing the role of a heat sink. Once equilibrium is reached, the heat remains at its maximum near the gold nanoholes in the metal part as well as in water near the upper surface, while it is as expected quite lower or null near the downward surface where $T=T_{r}$. The change in temperature upon illumination S1 at $980 \mathrm{~nm}$ (Fig. 2b), as measured by a photothermal-camera, shows a comparable temperature variation of about $25^{\circ} \mathrm{C}$.

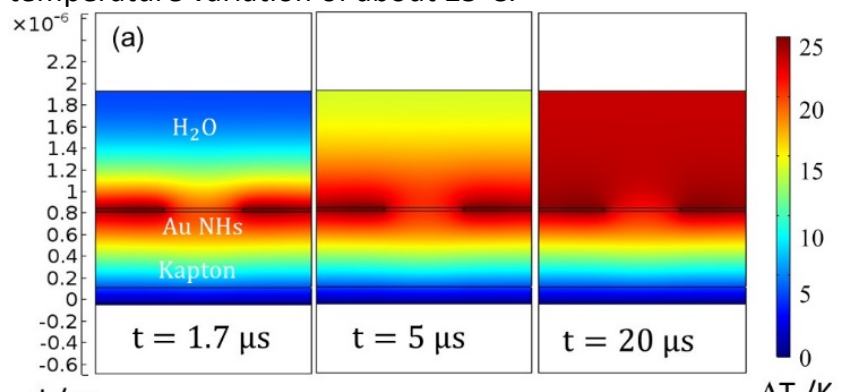

$\mathrm{d} / \mathrm{m}$

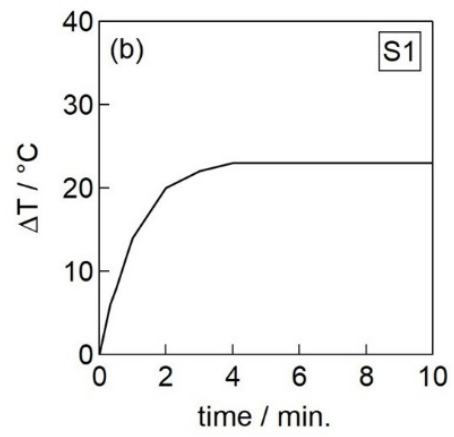

Fig 2. Plasmon driven temperature distribution map: (a) within a $\boldsymbol{z} \boldsymbol{x}$-cut plane (in the middle of the structure) at different instants of time, for an incident light intensity of $2 \mathrm{~W} \mathrm{~cm}^{-2}$. (b) Photothermal heating curves of S1 immersed in $0.1 \mathrm{M} \mathrm{NaOH}$ solution and exposed to laser light of $980 \mathrm{~nm}$ at 2 $\mathrm{W} \mathrm{cm}^{-2}$.

\subsubsection{Electrochemical component}

Under light irradiation at $980 \mathrm{~nm}$, the electrochemical charge transfer increases (Fig. 3a) with the current density rising linearly with the light intensity (Fig. $\mathbf{3 b}$ ) and an activation energy $\left(E_{\mathrm{a}}\right)$ of $13.7 \pm 0.4 \mathrm{~kJ} \mathrm{~mol}^{-1}$ was determined (Fig. 3c). It has been 
recently postulated that illumination intensity dependent photocatalytic activities lead to relevant mechanistic insights. ${ }^{12}$ While exponential relationships between photon-induced rates and illumination intensities have been assigned to mechanisms that are driven by a thermal process, a linear rate dependence on intensity is usually associated with an electron driven process, which corroborates our observations. To account for possible temperature-related effects, cyclic voltammograms were recorded in thermostated solutions with temperatures up to $50{ }^{\circ} \mathrm{C}$ (Fig. S1a), providing an activation energy of $E_{\mathrm{a}}=29.4 \pm$ $0.9 \mathrm{~kJ} \mathrm{~mol}^{-1}$ (Fig. 3c). The difference in $E_{\mathrm{a}}$ values is due to the plasmon-mediated effect and the generation of hot electrons, facilitating electron transfer and oxidation of ferrocenemethanol. In addition, a wavelength-dependent current is observed (Fig. S1b). Irradiation of S1 with a laser at $650 \mathrm{~nm}$ resulted in $E_{\mathrm{a}}=29.0 \pm 0.9 \mathrm{~kJ} \mathrm{~mol}^{-1}$, comparable to that of a heated solution and lower when compared to illumination at $980 \mathrm{~nm}$. This verifies experimentally the occurrence of plasmon mediated hot electron injection.
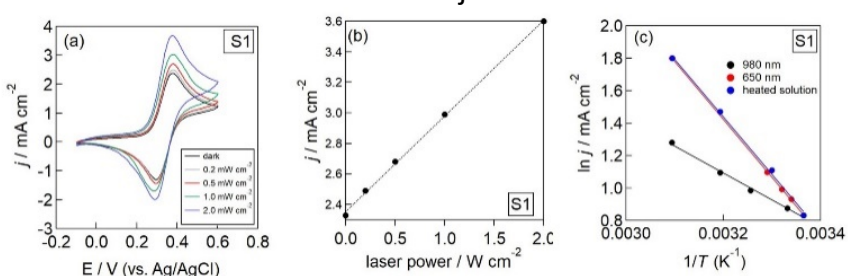

Fig. 3. Plasmon-enhanced electrochemical activity of nanoperforated gold thin film electrodes (S1): (a) Cyclic voltammograms (CVs) recorded in ferrocenemethanol $(10 \mathrm{mM}) / \mathrm{KCl}(0.1 \mathrm{M})$ with a scan rate of $100 \mathrm{mV}$ $\mathrm{s}^{-1}$ under light irradiation for $5 \mathrm{~min}$ at $980 \mathrm{~nm}$ with varying intensity. (b) Variation of the anodic peak current as a function of light intensity. (c) Arrhenius plots of the maximal current density derived from Fig. 3a.

\subsection{Plasmon-enhanced ORR activity}

The electrocatalytic activity of S1 towards ORR in alkaline condition (0.1 $\mathrm{M} \mathrm{NaOH}$ ) was investigated in the following. Several studies indicate that the first electron transfer to $\mathrm{O}_{2}$ determines the overall rate, being $\mathrm{O}_{2}+\mathrm{e}^{-} \rightarrow \mathrm{O}_{2}^{-}$in basic electrolytes. ${ }^{13}$ Note that using gold as electrode, the reaction depends on the surface atomic arrangement, being faster on $\mathrm{Au}(100)$ due to a lower coverage with absorbed $\mathrm{OH}$ in the region where the ORR occurs.

Plasmon-enhanced ORR activity was assessed by voltammetric measurements. Linear sweep voltammetry curves of $\mathrm{S} 1$ in $\mathrm{N}_{2}$-saturated and $\mathrm{O}_{2}$-saturated $0.1 \mathrm{NaOH}$ solution at an angular rotation rate of $100 \mathrm{rpm}$ and a scan rate of $25 \mathrm{mV} \mathrm{s}^{-1}$ are presented in Fig. 4a. An increase in the cathodic current is observed in the $\mathrm{O}_{2}$-saturated solution, suggesting that $\mathrm{S} 1$ has electrocatalytic activity for reducing oxygen. Irradiation with a laser light of $980 \mathrm{~nm}$ increases the current density related to oxygen reduction as a function of light intensity. The ORR onset potential, determined at a current density of $10 \mu \mathrm{A} \mathrm{cm}-2$ is 0.98 $\mathrm{V}$ (vs. RHE) at $0.5 \mathrm{~W} \mathrm{~cm}^{-2}, 1.02 \mathrm{~V}$ (vs. RHE) at $1 \mathrm{~W} \mathrm{~cm}^{-2}$ and 1.07

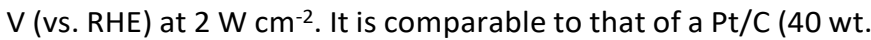
$\%$ ) electrode, experimentally determined as $1.07 \mathrm{~V}$ (vs. RHE) (Fig. 4b), and in excellent agreement with the onset potential reported by Perez et al., ${ }^{14} E_{\text {onset }}=1.07 \mathrm{~V}$ (vs. RHE). Moreover, the limiting current density upon irradiation at $2 \mathrm{~W} \mathrm{~cm}^{-2}(j=-$ $4.2 \mathrm{~mA} \mathrm{~cm}^{-2}$ ) is comparable to that of $\mathrm{Pt} / \mathrm{C}$ (40 wt.\%) under irradiation $\left(j=-3.9 \mathrm{~mA} \mathrm{~cm}^{-2}\right)$ and largely increased compared to that of Pt/C (10 wt.\%) $\left(j=-2.8 \mathrm{~mA} \mathrm{~cm}^{-2}\right)$ at $E=0.80 \mathrm{~V}$ (vs. RHE) bias. Performing ORR in a thermostated electrode (Fig. S2) results in much lower ORR currents even at $50^{\circ} \mathrm{C}(j=-3.1 \mathrm{~mA}$ $\mathrm{cm}^{-2}$ ). The performance of $\mathrm{S} 1$ under light irradiation is competitive to other electocatalysts reported in the literature (Table 1). ${ }^{15}$
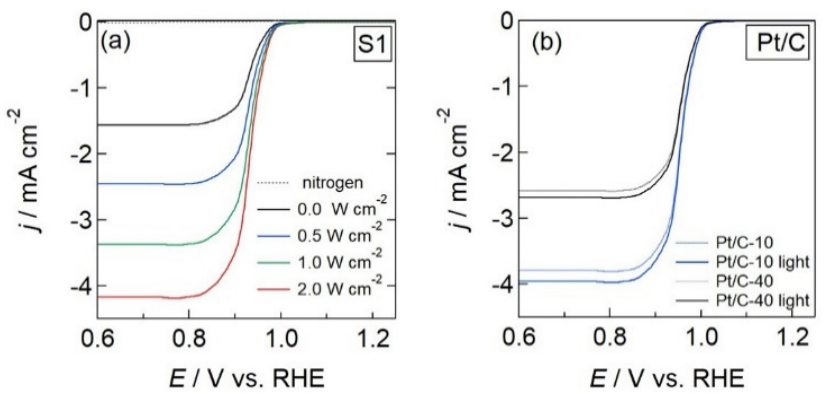

Fig. 4. Linear sweep voltammetry (LSV) curves at constant rotation speed of $1000 \mathrm{rpm}$ : (a)S1 in $\mathrm{N}_{2}$ (grey) and $\mathrm{O}_{2}$-saturated $0.1 \mathrm{M} \mathrm{NaOH}$ solution without and with laser illumination at $980 \mathrm{~nm}$ for $5 \mathrm{~min}$ at a power density of $0.5,1$ and $2 \mathrm{~W} \mathrm{~cm}^{-2}$. The scan rate is $25 \mathrm{mV} \mathrm{s}^{-1}$. (b) Pt/C (10 wt.\%) and Pt/C (40 wt.\%) in $\mathrm{O}_{2}$-saturated of $0.1 \mathrm{M} \mathrm{NaOH}$ solution without and with laser illumination at $980 \mathrm{~nm}$ for $5 \mathrm{~min}$ at $2 \mathrm{~W} \mathrm{~cm}$. Rotation speed: $1000 \mathrm{rpm}$; The scan rate is $25 \mathrm{mV} \mathrm{s}^{-1}$.

Table 1. ORR performance of precious metal-based catalysts in $\mathrm{O}_{2}$-saturated $\mathrm{NaOH}(0.1 \mathrm{M})$.

\begin{tabular}{|l|l|l|l|}
\hline Catalyst & $\begin{array}{l}E_{\text {onset }} \\
\text { (vs. RHE) }\end{array}$ & $\begin{array}{l}\text { Test } \\
\text { conditions }\end{array}$ & Ref. \\
\hline Nobel metal-based catalysts \\
\hline Pt/C & $1.07 \mathrm{~V}$ & alkaline/acidic & 14 \\
\hline Au NHs (980 nm) & $1.07 \mathrm{~V}$ & alkaline & $\begin{array}{l}\text { This } \\
\text { work }\end{array}$ \\
\hline Pd nanocubes & $1.00 \mathrm{~V}$ & alkaline /acidic & 16 \\
\hline Au (100) & $0.96 \mathrm{~V}$ & alkaline & 17 \\
\hline rGO/Au & $0.88 \mathrm{~V}$ & alkaline & 18 \\
\hline Au (111) & $0.84 \mathrm{~V}$ & alkaline & 16 \\
\hline Metal-free catalysts & 0.89 & alkaline & 19 \\
\hline $\mathrm{N}-$ doped graphene & 0.89 & 20 \\
\hline S- and N-doped CNT & 0.96 & alkaline \\
\hline Transition Metal-Carbon hydride \\
\hline Fe/N/C & 0.92 & alkaline & 21 \\
\hline Co/N/rGO & 0.93 & alkaline & 22 \\
\hline
\end{tabular}

\subsection{Mechanism}

To understand the origin of the current enhancement, the ORR reaction was performed at different solution temperatures up to $50^{\circ} \mathrm{C}$ (Fig. S2a). The temperature corresponds to the surface temperature as determined with a thermocouple attached to the sample when immersed in $0.1 \mathrm{M} \mathrm{NaOH}$ and irradiated with a $980 \mathrm{~nm}$ laser at $2.0 \mathrm{~W} \mathrm{~cm}^{-2}$ for $10 \mathrm{~min}$. We observed that the thermal heating effect on the ORR was weaker than the plasmonic effect.

To gain further evidence of the plasmon-mediated ORR, the activation energy $\left(E_{\mathrm{a}}\right)$ of ORR was determined under light illumination and compared to that of a thermostated solution Plots of $\ln j$ vs. $1 / T$ revealed a linear relationship in both cases (Fig. 5a). Using the Arrhenius equation, $E_{\mathrm{a}}$ under light irradiation was estimated as $22.2 \pm 0.2 \mathrm{~kJ} \mathrm{~mol}^{-1}$, while in dark it was $33.5 \pm$ 
$0.3 \mathrm{~kJ} \mathrm{~mol}^{-1}$. Plasmon-induced processes generated a significant higher ORR reactivity on S1 due to the formation of hot electrons.6, 9, 23 According to these results, the mechanism for the enhanced activity towards ORR under laser irradiation is as following (Fig. 5b): under laser excitation, localized surface plasmons are generated on the nanopatterned gold film electrode forming electron-hole pairs. The generated hot electrons can then follow different pathways: (i) recombination with the formed holes in the $\mathrm{Au} \mathrm{NHs}$ layer, (ii) direct electrochemical oxygen reduction in alkaline solutions on the Au NHs surface, or (iii) injection into the conducting band of the $\mathrm{Ti} / \mathrm{TiO}_{2}$ semiconductor adhesion layer, followed by oxygen reduction. In our case, both direct electrochemical reduction of oxygen and the injection of hot electrons via the Mott-Schottky barrier into the thin $\mathrm{Ti} / \mathrm{TiO}_{2}$ layer might occur, assuming that the electron injection is faster than the electron-hole recombination process. The fast generation and the injection of hot electrons at $980 \mathrm{~nm}$ affects the current density profile abruptly (Fig. 5c); irradiation at $680 \mathrm{~nm}$ results in no change in the current density profile, underlying a plasmon-driven effect occurring at $980 \mathrm{~nm}$.

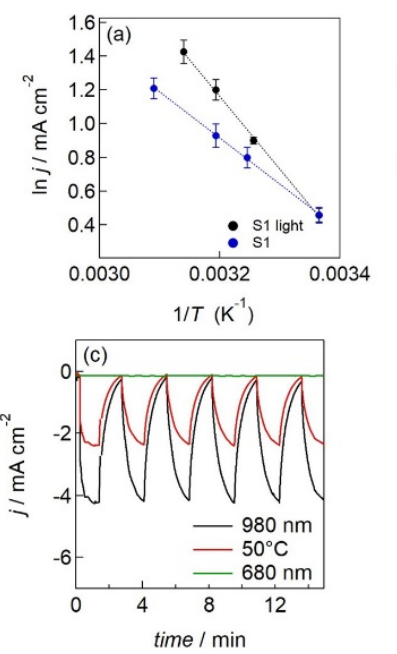

(b)

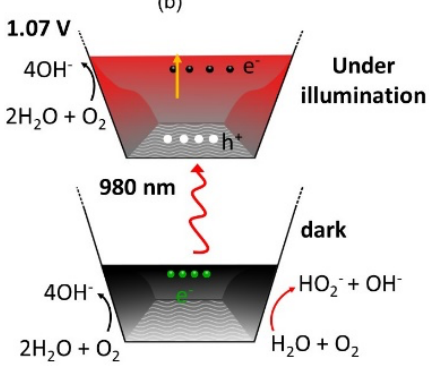

Au NHs electrode

Fig. 5. Mechanistic considerations: (a) Arrhenius plots under irradiation at $980 \mathrm{~nm}$ (black) and for a thermostated electrolyte (blue); (b) Schematics of the plasmon-enhanced ORR mechanism under basic conditions; (c) Current density - time curves with irradiation at $980 \mathrm{~nm}$ (black) at $2 \mathrm{~W} \mathrm{~cm}^{-2}, 680 \mathrm{~nm}$ at $2 \mathrm{~W} \mathrm{~cm}^{-2}$ (green) and with no illumination but thermostated at $50^{\circ} \mathrm{C}$ (red) when immersed in $0.1 \mathrm{M} \mathrm{NaOH}$ at +0.92 $\mathrm{V}$ (vs. RHE) bias.

Based on the polarization curves at $1000 \mathrm{rpm}$, the corresponding Tafel plots were estimated to evaluate the influence of light irradiation on the ORR kinetics (Fig. S3). The Tafel slopes for Pt/C (40 wt.\%) are $-72 \mathrm{mV} /$ decade (in dark)in accordance with other reports. ${ }^{14}$ Under light irradiation of $\mathrm{Pt} / \mathrm{C}$ (40 wt.\%) it drops to $-54 \mathrm{mV} /$ decade. In the case of Pt/C (10 wt.\%) Tafel slopes of $-100 \mathrm{mV} /$ decade were obtained and did not change upon irradiation. When illuminated at $980 \mathrm{~nm}, \mathrm{~S} 1$ exhibits a Tafel slope of $-44 \mathrm{mV} /$ decade, while in absence of illumination, a Tafel slope of $-377 \mathrm{mV} /$ decade was determined as expected to the low electrocatalytic activity of gold. The reduction in the Tafel slope suggests that the surface adsorption of oxygen is increasing and that oxygen bonds can be directly broken.
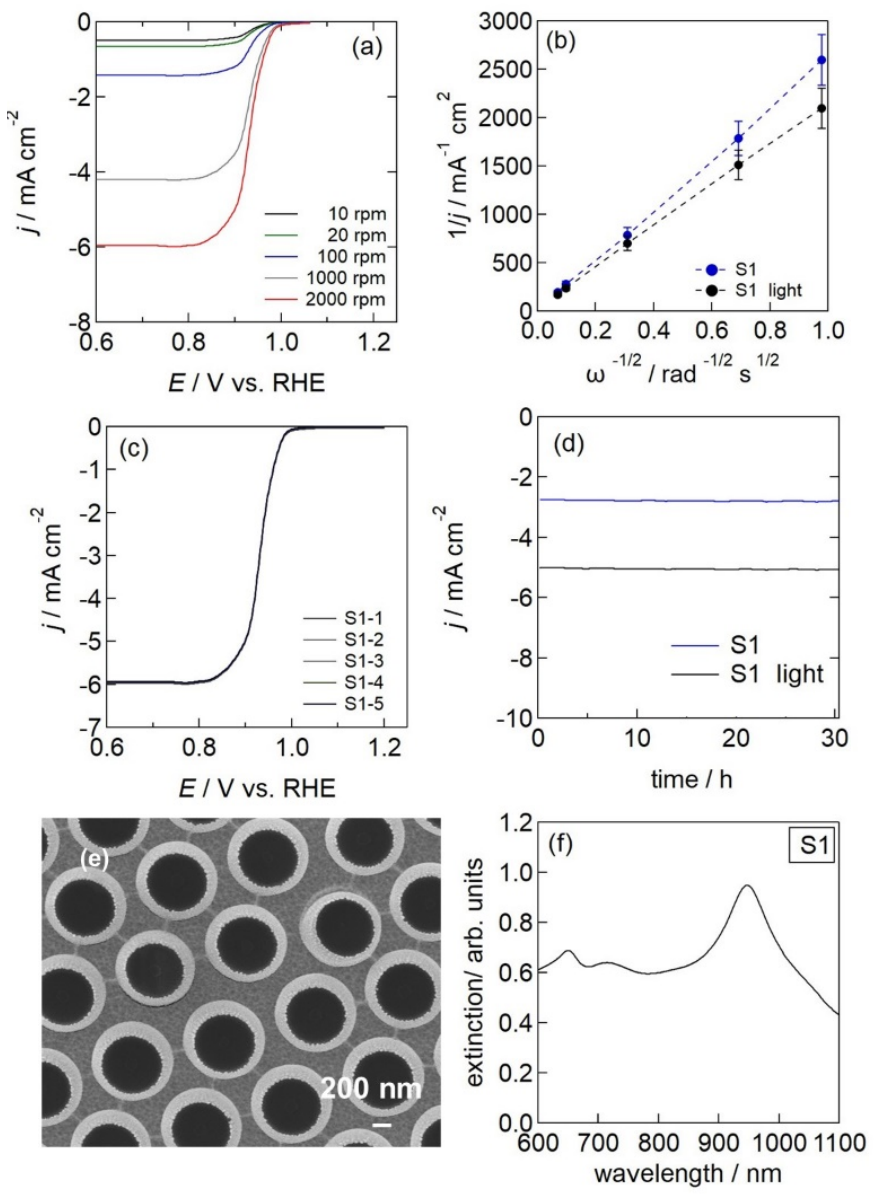

Fig. 6. Hydrodynamic conditions: (a) Current density vs. potential curves in $\mathrm{O}_{2}$-saturated $0.1 \mathrm{M} \mathrm{NaOH}$ solution recorded by angular rotation rates from 10 to $2000 \mathrm{rpm}$ under light irradiation at $980 \mathrm{~nm},(2$ $\mathrm{W} \mathrm{cm} \mathrm{cm}^{-2}$ ) at a scan rate of $25 \mathrm{mV} \mathrm{s}^{-1}$. (b) Koutecký-Levich plots under irradiation (data from panel a) and in dark (data from panel b to Fig. S2). (c) ORR polarization curves of five specimens in $\mathrm{O}_{2}$-saturated $0.1 \mathrm{M}$ $\mathrm{NaOH}$. (d) ORR stability tests at $E=+0.90 \mathrm{~V}$ without (blue) and with light irradiation at $2 \mathrm{~W} \mathrm{~cm}^{-2}$ (black) by following the change of the current density with time, (e) SEM image after ORR stability tests, (f) UV/Vis after ORR testing.

\subsection{Stability}

The ORR was found to be highly reproducible as seen in the overlapping LSV curves for 5 different electrodes (Figure 6c), while the long-time stability was assessed by current density time plots (Figure 6d). No significant decrease in the oxygen reduction current was observed over a period of $30 \mathrm{~h}$. Furthermore, the LSPR band as well as the morphology of the samples has not been altered after ORR stability tests. These results clearly confirm that nanoperforated gold thin film interfaces are suitable electrodes for the ORR reaction.

\section{Conclusion}

In conclusion, nanoperforated gold thin films irradiated at 980 $\mathrm{nm}\left(2 \mathrm{~W} \mathrm{~cm}{ }^{-2}\right)$ show electrocatalytic oxygen reduction in basic 
conditions comparable to that of commercial Pt/C electrodes. To underpin the mechanism of plasmonic enhancement of these interfaces, both ORR at different wavelengths and in electrolytes thermostated at different temperatures were investigated. The results indicated that hot electrons are generated from plasmon decay, which in turn facilitate the electrocatalytic oxygen reduction reaction. Using this experimental strategy, it was found that ORR under light irradiation proceeds via a $4 \mathrm{e}^{-}$pathway, while in dark it has a contribution from the $2 \mathrm{e}^{-}$side reaction. Plasmon-enhanced electrochemical reduction of oxygen to water on perforated gold nanohole electrodes represent another application of triple - plasmonic, chemical and catalytic - functional gold nanostructures. In general, this highly practical plasmoninduced hot electron enhancement method will be beneficial to energy and environmental applications and may open up avenues for the optical control of (electro)chemical reactions.

\section{Experimental}

\subsection{Materials}

Sodium hydroxide $(\mathrm{NaOH})$ and potassium hexacyanoferrate (II) $\left(\left[\mathrm{K}_{4} \mathrm{Fe}(\mathrm{CN})_{6}\right]\right)$ were purchased from Sigma-Aldrich and used as received.

Commercial E-TEK 10 wt.\% or 40 wt.\% Pt/C was used for making catalyst inks. The catalyst dispersion was prepared as follows: $5.0 \mathrm{mg}$ of $10 \mathrm{wt} . \% \mathrm{Pt} / \mathrm{C}$ was dispersed in $400 \mu \mathrm{L} \mathrm{EtOH}, 40 \mu \mathrm{L} 5$ wt.\% Nafion (Nafion ${ }^{\circledR}$ DE 520, Sigma-Aldrich) and $60 \mu \mathrm{L}$ deionized water by sonication for $15 \mathrm{~min}$. Then $10 \mu \mathrm{L}$ catalyst dispersion was transferred onto the glassy carbon disk by using a pipette and dried at room temperature. The catalyst loading on the electrode was $50 \mu \mathrm{g} \mathrm{Pt} / \mathrm{cm}^{-2}$ (10 wt.\% Pt/C) and $200 \mu \mathrm{g}$ $\mathrm{Pt} / \mathrm{cm}^{-2}$ (40 wt.\% Pt/C).

\subsection{Fabrication of gold nanoholes (Au NHs)}

Kapton interfaces were modified with gold nanoholes according to our previous work, ${ }^{24}$ with no Ti adhesion layer. In short, a monolayer of $1000 \mathrm{~nm}$ polystyrene beads (ThermoFisher Scientific) was deposited onto glass by self-assembly, followed by $\mathrm{SF}_{6}$ and oxygen plasma etching for $8 \mathrm{~min}$ (gas flow $2 \mathrm{sccm}$ and $30 \mathrm{sccm}$, respectively at $10 \mathrm{mTorr}$ chamber pressure) to reduce the particle size. The samples were then coated with $50 \mathrm{~nm} \mathrm{Au}$ at a constant deposition rate of $0.2 \AA \mathrm{s}^{-1}$ using physical vapor deposition. The beads on top of glass were removed by peeling the surface with Blue Low Tack tape (Semiconductor Equipment Corp.). The surfaces were copiously washed with acetone and dried under nitrogen flow. The arrays display holes of $630 \mathrm{~nm}$ average size and center-to-center spacing of $980 \mathrm{~nm}$.

\subsection{Characterization}

Scanning electron microscopy (SEM) images were obtained using an electron microscope ULTRA 55 (Zeiss) equipped with a thermal field emission emitter, three different detectors (EsB detector with filter grid, high efficiency in-lens SE detector, Everhart-Thornley Secondary Electron detector). The UV/vis absorption spectra were recorded using a Perkin Elmer Lambda UV-Vis 950 spectrophotometer

\subsection{Electrochemical experiments}

Electrochemical measurements were performed with a potentiostat/galvanostat (Metrohm Autolab) electrochemical test station in a standard three-electrode system with the plasmonic electrode (or Pt/C coated electrode) as the working electrode, a carbon plate as the counter electrode and an $\mathrm{Ag} / \mathrm{AgCl}(3.5 \mathrm{M} \mathrm{KCl})$ electrode as the reference electrode. The measured potentials were converted with respect to the reversible hydrogen electrode $(\mathrm{RHE})$ according to $E_{\mathrm{RHE}}=E_{\mathrm{Ag} / \mathrm{AgCl}}$

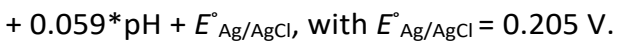

Cyclic voltammetry (CV) and linear sweep voltammetry (LSV) measurements were performed in either $\mathrm{K}_{4} \mathrm{Fe}(\mathrm{CN})_{6} 10 \mathrm{mM} / 0.1$ $\mathrm{M} \mathrm{KCl}$ or $0.1 \mathrm{M} \mathrm{NaOH}$ solution at the scan rate of 25 or $100 \mathrm{mV}$ $\mathrm{s}^{-1}$.

The electrochemically active surface area $(A)$ in the electrochemical cell was derived from the linear plot of the peak current as a function of the square root of the scan rate according to equation $A=s /\left(268600 \times n^{3 / 2} \times D_{f}^{1 / 2} \times c_{f}\right)$, where $n$ is the number of electrons transferred in the redox event $(n=1)$, $D_{f}$ is the diffusion coefficient of ferrocenemethanol $\left(7.5 \times 10^{-6}\right.$ $\left.\mathrm{cm}^{2} \mathrm{~s}^{-1}\right), c_{f}$ is the concentration of ferrocenemethanol $(0.0001$ $\mathrm{mol} \mathrm{cm}-3$ ), and $s$ is the slope of the linear fit to the data. The exposed area of the working electrode was $0.75 \mathrm{~cm}^{2}$.

For plasmon-enhanced electrocatalysis, the electrode was illuminated with light from a continuous wave laser (980 Gbox model, Fournier Medical Solution) with a laser power output between 0.5 and $2 \mathrm{~W} \mathrm{~cm}^{-2}$. Irradiation at $680 \mathrm{~nm}$ was performed using a laser from Phototech. The temperature changes were captured by an infrared camera (Thermovision A40) and treated using ThermaCam Researcher Pro 2.9 software.

Rotating Disk Electrode (RDE) measurements were performed on an electrode holder of $0.8 \mathrm{~cm}$ in diameter and carried out in $0.1 \mathrm{M} \mathrm{NaOH}$ using a modulated speed rotator (Radiometer Analytical) at a scan rate of $25 \mathrm{mV} \mathrm{s}^{-1}$. Oxygen was used to purge the solution to achieve an oxygen-saturated electrolyte. The laser light was directed onto the rotating disk part of the electrode. All measurements were repeated at least four times. The number of electrons transferred per $\mathrm{O}_{2}$ molecule in the ORR for $\mathrm{Pt} / \mathrm{C}$ and $\mathrm{Au} \mathrm{NHs}$ with and without light irradiation was calculated by the Koutecký-Levich equation applied to the ORR curves obtained at different rotation speeds (rpm).

\subsection{Optical Modeling}

The electric field distribution within the plasmonic structure was computed with Comsol Multiphysics. Floquet periodic boundary conditions were applied along $x$ and $y$ directions and perfectly matched layers (PMLs) were added on top and the bottom of the system to avoid reflections. The frequency dependent complex dielectric constant of gold metal is described by the Lorentz Drude model as 


$$
\varepsilon_{r}(\omega)=\varepsilon_{\infty}+\sum_{m=0}^{M} \frac{f_{m} \omega_{p}^{2}}{\omega_{m}^{2}-\omega^{2}+j \omega \Gamma_{m}}
$$

where $\varepsilon_{\infty}, \omega_{p}, \omega_{m}, f_{m}$ and $\Gamma_{m}$ are the relative permittivity at infinite frequency, the plasma frequency, the resonance frequency strength and the damping frequency of $m^{\text {th }}$ oscillator. The values of these parameters are taken from Ref. ${ }^{25}$, while the refractive indexes of the remaining materials are set to 1.5 for Kapton and 1.33 for water, the incidence medium set on top of Au NHs. The incident light wave used for excitation is linearly polarized such that the associated electric field is set along the $x$-axis, which is parallel to the longest length of the unit cell.

\subsection{Thermal effect modeling}

A 3D heat transfer model has been set based on finite element method (using Comsol Multiphysics). Numerically, we solve the transient heat transfer equation which reads as follows:

$$
C_{s} \rho \frac{\partial T}{\partial t}+\nabla \cdot(-k \nabla T)=Q_{p}
$$

where, $T$ is the temperature in the system (time and space dependent), $C_{s}, \rho$ and $k$ are respectively the heat capacity, density and thermal conductivity of the considered material (within the system). On the right side of the equation, $Q_{p}$, is the heat source driving the thermal effects in space and time in the structure. This quantity arouses out of the resistive heating occurring in the gold metal parts near and around the $\mathrm{NHs}$ where the localized surface plasmon is excited; it is given by:

$$
Q_{p}=\frac{1}{2} \varepsilon_{0} \omega \operatorname{Im}\left(\varepsilon_{r}\right)|\vec{E}|^{2}
$$

$\varepsilon_{r}$, is the gold relative permittivity whose expression is set based on Lorentz Drude model (see down below), $\varepsilon_{0}$ is vacuum permittivity, $\omega$ and $\vec{E}$ are the angular frequency and the electric field of the upcoming light wave. The incident light intensity is maintained fixed throught out the simulation at $I_{0}=2 \mathrm{~W} / \mu \mathrm{m}^{2}$. The applied boundary conditions for the thermodynamic study are such as: i) along $x$ and $y$ directions we set periodic conditions on the boundaries planes (perpendicular to $x$ and $y$ ) of the studied unit cell, that is, the horizontal heat flux normal to these planes is zero, ii) on the downside boundary the temperature of Kapton is fixed equal to the ambient temperature $T_{r}=293.15 \mathrm{~K}$, and iii) finally on the upper boundary which is water surface in contact with air, we set the outward conductive heat flux equal to convective heat flux into air plus the water surface heat radiation. This latter boundary condition can be written as:

$$
\vec{n} .(-k \nabla T)=h\left(T-T_{r}\right)+\varepsilon \sigma\left(T^{4}-T_{r}^{4}\right)
$$

$\vec{n}$, is the outward normal vector of the boundary surface (water surface), $h=5 \mathrm{~W} / \mathrm{m}^{2} \mathrm{~K}$ is convective heat transfer coefficient of air and $\varepsilon \approx 0.96$ and $\sigma=5.67 .10^{-8} \mathrm{~W} / \mathrm{m}^{2} \mathrm{~K}^{2}$ refer to the water surface emissivity and to the Stefan-Boltzmann constant, respectively. We considered the fact that bulk gold material thermal conductivity is bigger as compared with the one corresponding to nanosize gold films. As a matter of fact, this is mentioned in the work of Chen et al. ${ }^{26}$ where it is indicated that gold conductivity falls from $317 \mathrm{~W} / \mathrm{k} . \mathrm{m}$ for a bulk material down to $110 \mathrm{~W} / \mathrm{k} . \mathrm{m}$ for a $\sim 30 \mathrm{~nm}$ gold metal film.

\section{Conflicts of interest}

There are no conflicts to declare.

\section{Acknowledgements}

Financial supports from the Centre National de la Recherche Scientifique (CNRS), the University of Lille and the Hauts-deFrance region via the dispositif STIMUIE (Soutien aux travaux interdisciplinaires) 2019 are acknowledged. A.G.M.S. thanks to FAPESP for his postdoctoral fellowship (grant number 2018/25567-7). S.M. is a Research Associate of the Belgian F.R.S. - FNRS and acknowledges financial support via the SELFPHON project. T. Nait Saada thanks the French embassy in Algeria and the Algerian Ministry of Higher Education and Scientific Research (M.E.S.R.S) for a PROFAS B+ 2018 grant.

\section{References}

1. L. Mascaretti, A. Dutta, Š. Kment, V. M. Shalaev, A. Boltasseva, R. Zbořil and A. Naldoni, Adv. Mater., 2019, 31, 1805513.

2. M. Shao, Q. Chang, J. P. Dodelet and R. Chenitz, Chem Rev. , 2016, 116, 3594-3657.

3. J. Greeley, I. E. Stephens, A. S. Bondarenko, T. P. Johansson, H. A. Hansen, T. F. Jaramillo, J. Rossmeisl, I. Chorkendorff and J. K. Nørskov, Nat. Chem., 2009, 1, 552-556.

4. S. S. Wang, L. Jiao, Y. Qian, W.-C. Hu, G.-Y. Xu, C. Wang and H.-L. Jiang, Angew. Chem., Int. Ed., 2019, 58, 10713-10717.

5. C. H. Choi, K.Chung, T.-T Nguyen and D.H. Kim, ACS Energy Lett., 2018, 3, 1415-1433.

6. F. Shi, J. He, B. Zhang, J. Peng, Y. Ma, W. Chen, F. Li, Y. Qin, Y. Liu, W. Shang, P. Tao, C. Song, T. Deng, X. Qian, J. Ye and J. Wu, Nano Lett., 2019, 192, 1371-1378.

7. S.-C. Lin, C.-S. Hsu, S.-Y. Chiu, T.-Y. Liao and H. M. Chen, J. Am. Chem. Soc., 2017, 139, 2224-2233.

8. D. Chen, R. Zhang, R. Wang, L. Dal Negro and S. D. Minteer, J. Electrochem. Soc., 2016, 163, H1132-H1135.

9. C. Wang, X.-G. Nie, Y. Shi, Y. Zhou, J.-J. Xu, X.-H. Xia and H.Y. Chen, ACS Nano, 2017, 11, 5897-5907.

10. C. Wang, X.-P. Zhao, Q.-Y. Xu, X.-G. Nie, M. R. Younis, W.-Y. Liu and X.-H. Xia, ACS App. Nano Mater., 2018, 1, 58055811.

11. K. Cheng, S. Wang, Z. Cui, Q. Li and S. Dai, App. Phys. Lett. , 2012, 100, 253101.

12. M. J. Kale, T. Avanesian and P. Christopher, ACS Catal. , 2014, 4, 116-128.

13. P. Quaino, N. B. Luque, R. Nazmutdinov, E. Santos and W. Schmickler, Angew. Chem., Int. Ed., 2012, 51, 12997.

14. J. Perez, E. R. Gonzalez and E. A. Ticianelli, Electrochim. Acta, 1998, 44, 1329-1339.

15. X. Ge, A. Sumboja, D. Wuu, T. An, B. Li, F. W. T. Goh, T. S. A. Hor, Y. Zong and Z. Liu, ACS Catal., 2015, 5, 4643-4667.

16. H. Eriskon, A. Sarapuu, N. Alexeyeva, K. Tammeveski, J. Solla-Gullon and J. M. Feliu, Electrochem. Acta, 2012, 59, 329. 
17. R. R. Adžić, S. Śtrbac and N. A. Anastasijević, Mater. Chem. Phys., 1989, 22, 349.

18. H. Yin, H. Tang, D. Wang, Y. Gao and Z. Tang, ACS Nano, 2012, 6, 8288.

19. H. P. Cong, P. Wang, M. Gong and S.-H. Yu, Nano Energy, 2014, 3, 55-63.

20. Q. Shi, F. Peng, S. Liao, H. Wang, H. Yu, Z. Liu, B. Zhang and S. Dangsheng, J. Mater. Chem. A, 2013, 1, 14853-14857.

21. L. Lin, Q. Zhu and A. W. Xu, J. Am. Chem. Soc., 2014, 136, 11027.

22. B. Zheng, J. Wang, F.-B. Wang and X.-H. Xia, J. Mater. Chem. A, 2014, 2, 9079-9084.

23. S. Mukherjee, F. Libisch, N. Large, P. Neumann, L. V. Brown, J. Cheng, J. Britt Lassite, E. A. A. Carte, P. Nordlander and N. J. Halas, Nano. Lett., 2013, 13, 240-247.

24. C. Li, R. Ye, J. Bouckaert, A. Zurituza, D. Dridier, Dumych, S. Paryzhak, V. Viovk, R. O. Bilyy, S. Melinte, M. Li and R. Boukherroub, ACS Appl. Mater. Interfacs, 2017, 9, 3666536674.

25. A. D. Rakic, A. B. Djurišić, J. M. Elazar and M. M. L, Appl. Opt., 1998, 37, 5271-5283.

26. X. Cheng, Y. Chen, M. Yan and M. Qiu, ACS Nano, 2012, 6, 2550-2557. 